Revista del Centro de Investigación de la Universidad La Salle

Vol. 14, No. 53, Enero-Junio, 2020: 265-290

DOI: http://doi.org/10.26457/recein.v14i53.2683

\title{
Análisis de los efectos del COVID-19 en la economía mexicana
}

\section{Analysis of the effects of COVID-19 on the Mexican economy}

\author{
Cesaire Chiatchoua ${ }^{1}$ \\ Universidad La Salle México (México) \\ Carmen Lozano \\ Universidad La Salle México (México) \\ Jesús Macías-Durán \\ CINVESTAV, IPN (México)
}

Recibido: 05 de junio de 2020

Aceptado: 12 de agosto de 2020

Publicado: 21 de septiembre de 2020

\section{Resumen}

El impacto económico por la pandemia por coronavirus (COVID-19) ha empezado a sentirse en todo el mundo y México no es ajeno a este panorama desalentador. Es necesario proponer soluciones para mitigar los efectos de esta crisis. El gobierno mexicano ha implementado medidas como son el acopio de recursos para apoyar la infraestructura de salud, el trabajo conjunto con las fuerzas armadas y su infraestructura sanitaria, el incremento al presupuesto

\footnotetext{
${ }^{1}$ Email: cesaire.chiatchoua@lasalle.mx
}

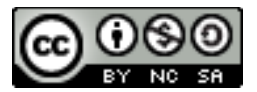

Revista del Centro de Investigación. Universidad La Salle por Dirección de Posgrado e Investigación. Universidad La Salle Ciudad de México se distribuye bajo una Licencia Creative Commons Atribución- 
para fortalecer el blindaje de los programas sociales en 622 mil 556 millones de pesos, el otorgamiento de 2 millones 100 mil créditos a trabajadores y 3 millones de créditos a la población más necesitada y, a pesar de esto, la situación ha sido inestable. Con base en la aplicación de los modelos promedios móviles autorregresivos integrados (ARIMA) se pronosticó el comportamiento de factores económicos tales como el PIB, la inflación, las remesas, el Índice de Consumo Mensual (ICM) y el Índice Nacional de Precio al Consumidor (INPC) con la finalidad de generar propuestas que fortalezcan a la economía mexicana. Para el análisis se utilizaron datos de fuentes como el Banco mundial, el Instituto Nacional de Estadística y Geografía y Banco de México. Los resultados muestran que el INPC disminuirá al continuar el año, el desempleo seguirá en aumento, la tasa de inflación se ubicará entre 3.0 y 3.5 al cierre del año 2020, las remesas continuarán aparentemente constantes y el ICM muestra tendencia positiva a corto plazo para situarse cercano al valor 40. Por lo tanto, es recomendable que el gobierno aumente el presupuesto, así como la anticipación y organización ante este tipo de catástrofes.

Palabras clave: ARIMA, factores económicos, pandemia, pronóstico. 


\section{Abstract}

The economic impact due to the COVID-19 pandemic is now becoming noticeable around the world and Mexico is not an exception to this discouraging picture. It is necessary to propose solutions to mitigate the effects of this crisis. The Mexican government has implemented measures to alleviate the situation, such as the collection of resources to support heath infrastructure, the collaborative work with the armed forces and their sanitary infrastructure, increasing the budget to strengthen social programs to 622 thousand 556 millions Mexican pesos, granting 2 millions100 thousands of credits to workers and 3 millions of credits to the most vulnerable population and, despite that, the situation has been unstable. In this work, the economic factors such that Gross Domestic Product (GDP), Inflation Rate, remittances, Monthly Purchase Index (MPI) and National Consumer Price Index (NCPI), for the closure of 2020 using ARIMA models were estimated, with the intention of providing solutions to fortify Mexico's economy. To carry out this analysis, sources such as the World Bank, National Institute of Statistic and Geography, and the Bank of Mexico were consulted. The results show that the NCPI will decrease, unemployment will continue to rise, the inflation rate will place itself between 3.0 and 3.5, remittances will remain apparently stable and the MPI shows a positive trend to locate itself close to value 40 by the end of the year. Therefore, it is recommended that the government increase the budget, as well as the anticipation and organization of these kinds of these catastrophes

Keywords: ARIMA, economic factors, pandemic, forecast. 


\section{Introducción}

El mundo ya ha sufrido experiencias relacionadas con enfermedades que han puesto en alerta a la población como lo es el síndrome respiratorio agudo grave (SARS, por sus siglas en inglés) y la influenza AH1N1. Es natural abordar estas enfermedades desde el enfoque económico. El SARS se extendió en al menos 28 países, incluidos Australia, Brasil, Canadá, Sudáfrica, España y los Estados Unidos y su impacto económico se estima en 40 mil millones de dólares (Lee y McKibbin, 2004a). Actualmente, existen estudios que proporcionan análisis más detallados de los costos económicos. A decir, Lee y McKibbin (2004b), proporcionaron una evaluación de los impactos económicos del SARS. En este documento los autores abordaron los impactos en el comportamiento del consumo y la inversión a través de cambios en el costo y el riesgo de hacer negocios.

Por su parte, McKibbin, Anu y Sidorenko (2006) exploraron las implicaciones de un brote de influenza pandémica en la economía global a través de una variedad de escenarios, encontrando que incluso una pandemia leve tiene consecuencias significativas para la producción económica mundial. En este mismo trabajo se estimó que el costo de esta pandemia fue de 4.4 miles de millones de dólares.

Con relación a la pandemia actual, existen estudios previos que intentan describir los impactos macroeconómicos a nivel global. A priori, surgen diversos estudios cualitativos como una respuesta rápida a este problema. Por ejemplo, Kuckertz, Brändle, Gaudig, Hinderer, Morales-Reyes, Prochotta, Steinbrink y Berger (2020) ilustran cómo los empresarios están lidiando con los efectos de la crisis y lo que están haciendo para proteger sus empresas. Por su parte, Barua (2020), ilustra los probables impactos utilizando un modelo macroeconómico. Sin embargo, la realización de estudios cuantitativos en este campo se ve limitada por los datos disponibles y la singularidad de esta pandemia.

De lo anterior señalado surge la siguiente pregunta ¿Cuál es el escenario de la economía mexicana para los próximos meses? Para responder a esta interrogante se plantea el siguiente objetivo: pronosticar el comportamiento de factores económicos como el PIB, la inflación, las remesas, el Índice de Consumo Mensual (ICM), el Índice Nacional de Precios al Consumidor (INPC) con la finalidad de generar propuestas que fortalezcan a la economía mexicana. Partiendo del hecho de que las políticas implementadas para disminuir el impacto 
económico del COVID-19 no han mostrado un efecto positivo, derivado de la inmovilidad convocada por el gobierno mexicano, el panorama para la recuperación de la economía nacional es desalentador.

La mayoría de los estudios sobre los efectos económicos de esta epidemia se centran en los costos médicos o los ingresos perdidos por la mortalidad, ambos asociados con la enfermedad y son pocos los centrados en el impacto macroeconómico para las naciones. Si bien es un poco apresurado hablar de medir los efectos económicos del COVID-19 en la economía nacional, es pertinente comprender el panorama internacional para tener una visión del efecto que podría tener en México. Es así como se espera que esta investigación pueda contribuir a hallar soluciones para disminuir los efectos económicos de la pandemia del COVID-19 en México.

\section{El Panorama del COVID-19}

Desde que la Organización Mundial de la Salud (OMS, 2020) declaró el brote como una pandemia, muchos países cerraron fronteras para tratar de evitar los contagios; redujeron los flujos de bienes y servicio y la mayoría de los negocios y empresas cerraron de manera temporal. El impacto económico ha empezado a sentirse en todo el mundo.

La expectativa de corto plazo para la economía mundial muestra efectos negativos, los mercados, así como los índices bursátiles mundiales muestran efectos negativos (Baldwin y di Mauro, 2020). A manera de ilustración, durante febrero y marzo Italia reportó su mayor caída del mercado accionario con 41\%, China 13\%, mientras que el Dow Jones y la Bolsa Mexicana de Valores presentaron caídas del 33\% y 22\%, respectivamente.

A nivel mundial se indican caídas anuales del PIB del 3.8\% en los Estados Unidos, el $9 \%$ en la zona del euro y el $2.1 \%$ en el Japón, y una desaceleración en China que la llevaría a un crecimiento de sólo 3\%. Este es un escenario de recesión mundial; más aún, las economías podrían incluso enfrentarse a una contracción de mediano plazo sin una rápida recuperación (CEPAL, 2020). América Latina y el Caribe enfrentan la crisis sanitaria del COVID-19 con un enorme desafío social cuyos efectos económicos podrían llevar a la región a una nueva década perdida.

La respuesta de países actores como China, Estados Unidos, Japón, Alemania, Gran Bretaña, Francia e Italia puede ser determinante en la economía global. Según Baldwin y di 
Mauro (2020, como se citó en Barua, 2020) estos países comparten el $60 \%$ de la oferta del PIB mundial, el $65 \%$ de la fabricación mundial y el $41 \%$ exportaciones mundiales de manufacturas.

Puesto que estas economías ahora se ven gravemente afectadas es preocupante para países de América Latina cuya población tienen marcadas desigualdades sociales. Powell (2020) señala que la tasa de crecimiento para la región en 2020 se sitúa en un rango entre el $-1.8 \%$ y el $-5.5 \%$, los choques fuerte y severo presentan rangos Holadel $-3.0 \%$ al $-3.9 \%$. En otros sectores, comenta la ONU (2020) que América Central tendrá una caída del PIB de $2.3 \%$, afectada por el descenso del turismo y de las remesas; mientras que el Caribe se contraerá en un $2.5 \%$, debido a la reducción de la demanda de servicios turísticos.

La pandemia del COVID-19 es uno de los desafíos más importantes que ha enfrentado la humanidad en tiempos recientes. Todavía se desconoce cuál podrá ser su costo total en vidas humanas. De manera simultánea a la pérdida de vidas y a la profunda crisis de salud, el mundo está siendo testigo de un colapso económico que impactará de manera severa el bienestar de grandes segmentos de la población durante los próximos años (Hevia y Neumeyer, 2020).

En México la pandemia también afectó diversos sectores de la economía. Por ejemplo, entre marzo y abril se perdieron más de medio millón de empleos (Flores, 2020); Banco de México (2020a) pronosticó una caída de 9\% del PIB durante los últimos cuatro meses. Las medidas de cuarentena adoptadas por el gobierno mexicano para hacer frente a la pandemia han reducido drásticamente la producción nacional. Aunado a eso, la caída de las exportaciones e importaciones han encarecido los productos en los mercados, llevando a un incremento de la inflación. El sector turismo se vio fuertemente afectado, sin mencionar una disminución de las remesas y un incremento de la violencia, tanto en los hogares como en el crimen organizado (Expansión, 2020).

La Figura 1 muestra la evolución del PIB de 1980 a 2020. Se observa una caída lenta a partir de 2020. En un comunicado de prensa, el INEGI (2020d) afirmó que el PIB del primer Trimestre de 2020 disminuyó 1.6\% frente al trimestre previo. Por componentes, el PIB de las Actividades Secundarias y Terciarias descendió 1.4\% de manera individual, mientras que el de las Actividades Primarias aumentó $0.5 \%$ en el periodo señalado con relación al trimestre 
precedente. Finalmente, en El Economista (2020a), se menciona que Citibanamex estima que el PIB se contraiga hasta $9.0 \%$ en el 2020 como consecuencia principalmente de la pandemia.

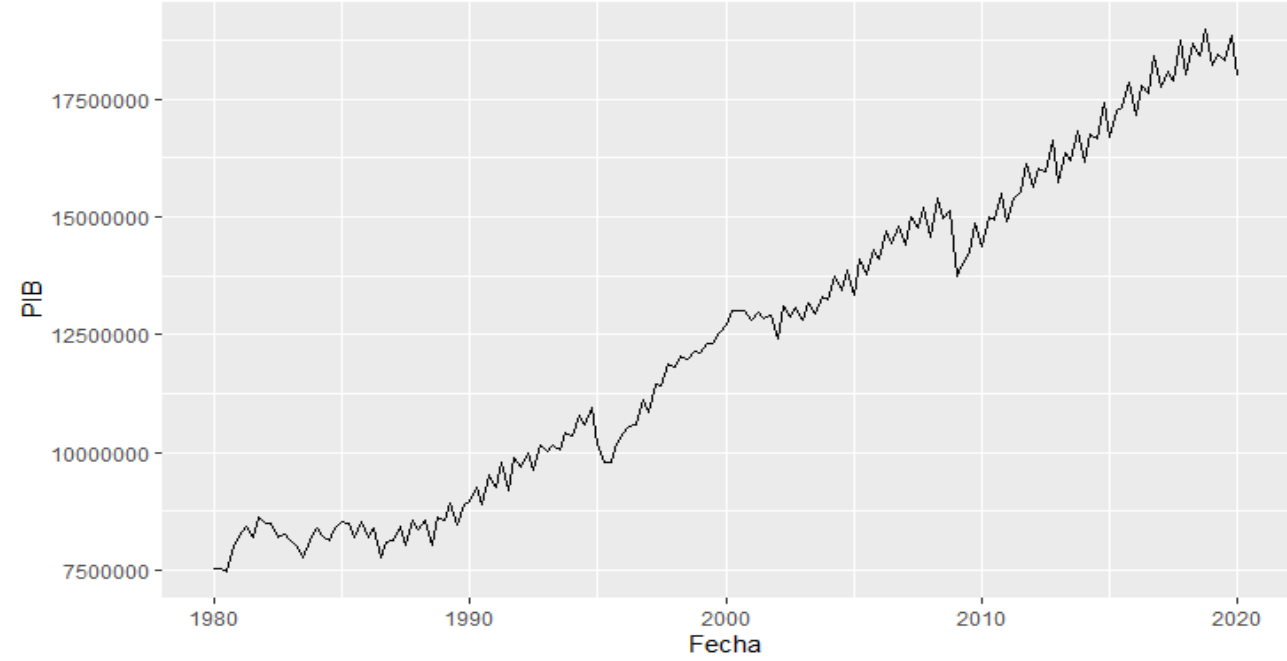

Figura 1. Producto Interno Bruto en México trimestral 1980-2020.Fuente. Elaboración propia con datos de Banco de México (2020a)

La Figura 2 muestra el índice de precios al consumidor. Entre enero y febrero se observa un crecimiento constante, las primeras caídas y recuperación se observan entre final de febrero e inicio de marzo. La caída prolongada se muestra a partir del 6 de marzo hasta el 25. Después de este período se observan movimientos de alza y baja hasta el 6 de abril.

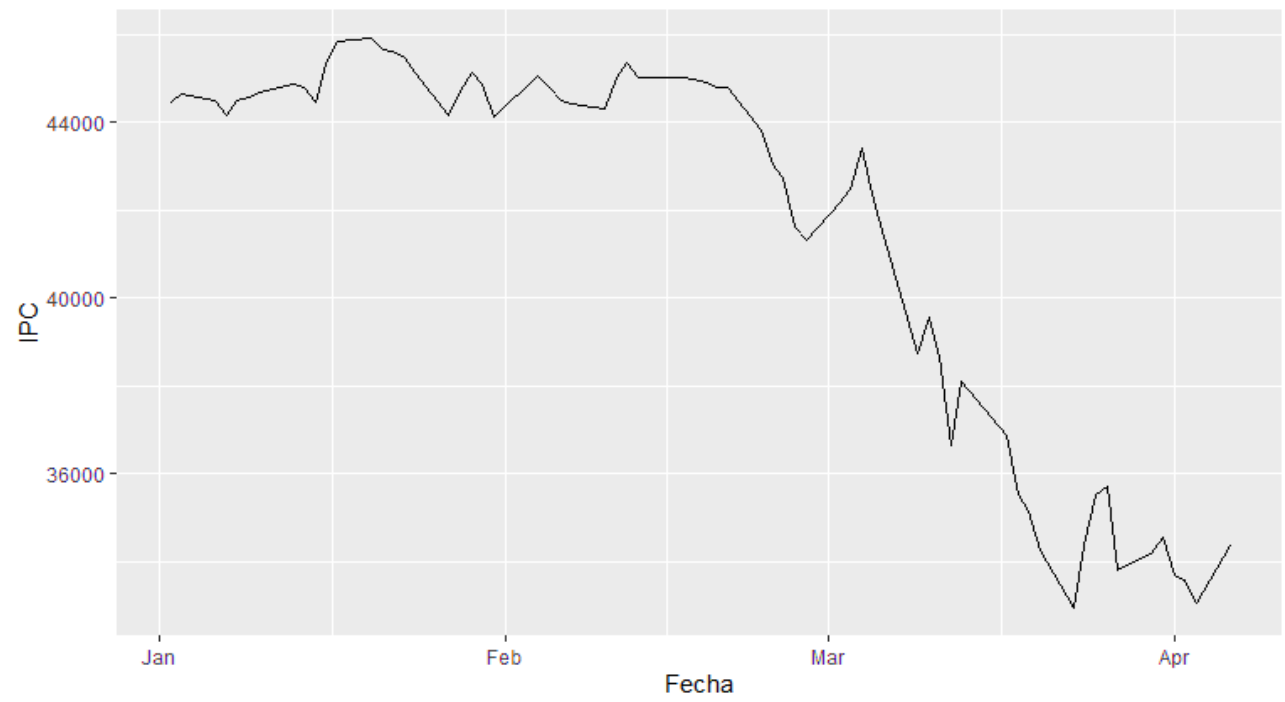

Figura 2. Índice de precio al consumidor durante el primer cuatrimestre del año 2020. Fuente: Elaboración propia con datos de Statistic (2020a). 
La Figura 3 muestra la evolución del tipo de cambio del peso mexicano frente al dólar. La reducción de las exportaciones netas mexicanas provocado por la pandemia del COVID-19 y la caída del precio del petróleo derivado de la disputa entre la republica de Rusia y Arabia Saudita, han llevado a la depreciación del peso frente al dólar. El 2 de febrero el dólar se cotizaba en 18.84 pesos, mientras que para el martes 24 de marzo del año en curso a 25.02 pesos por dólar. La apertura progresiva de algunos negocios esenciales y otros como el sector automotriz, así como el aumento de la movilidad dentro del país, han favorecido a la apreciación de la moneda de tal manera que para el lunes 01 de junio el valor bajó a 22.01 pesos según Banco de México (2020b).

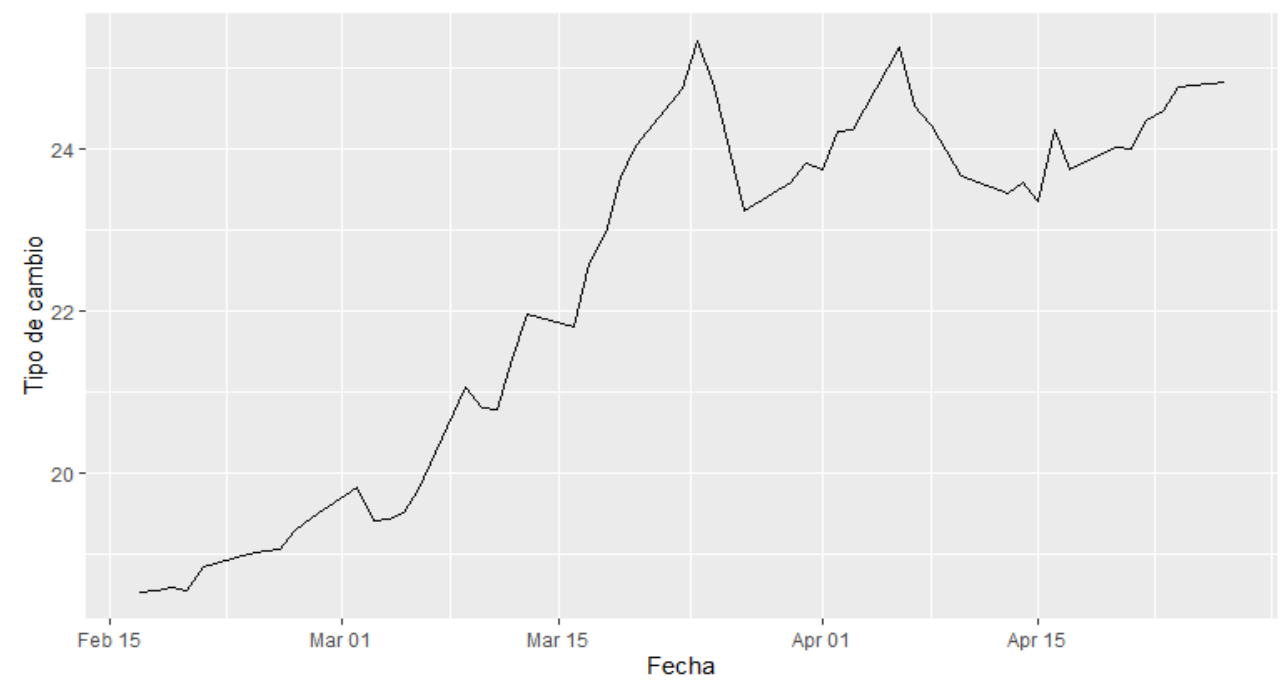

Figura 3. Tasa de cambio peso/dólar entre febrero y el 17 de abril 2020. Fuente: Elaboración propia con datos de Statista (2020b)

La Figura 4 presenta la tasa de desocupación en México. Se observa que de febrero a marzo se perdieron alrededor de 130,593 empleos (INEGI, 2020b). Este incremento del desempleo es inevitable debido a que mucha gente se ha quedado sin trabajo durante la contingencia, además de que tampoco lo está buscando gracias al aislamiento. Para el mes de abril, la situación se vuelve más difícil dado un estancamiento de la actividad económica en varios sectores como el turismo, los pequeños negocios, el transporte. INEGI (2020c) señala que se perdieron más de 555 mil empleos. 


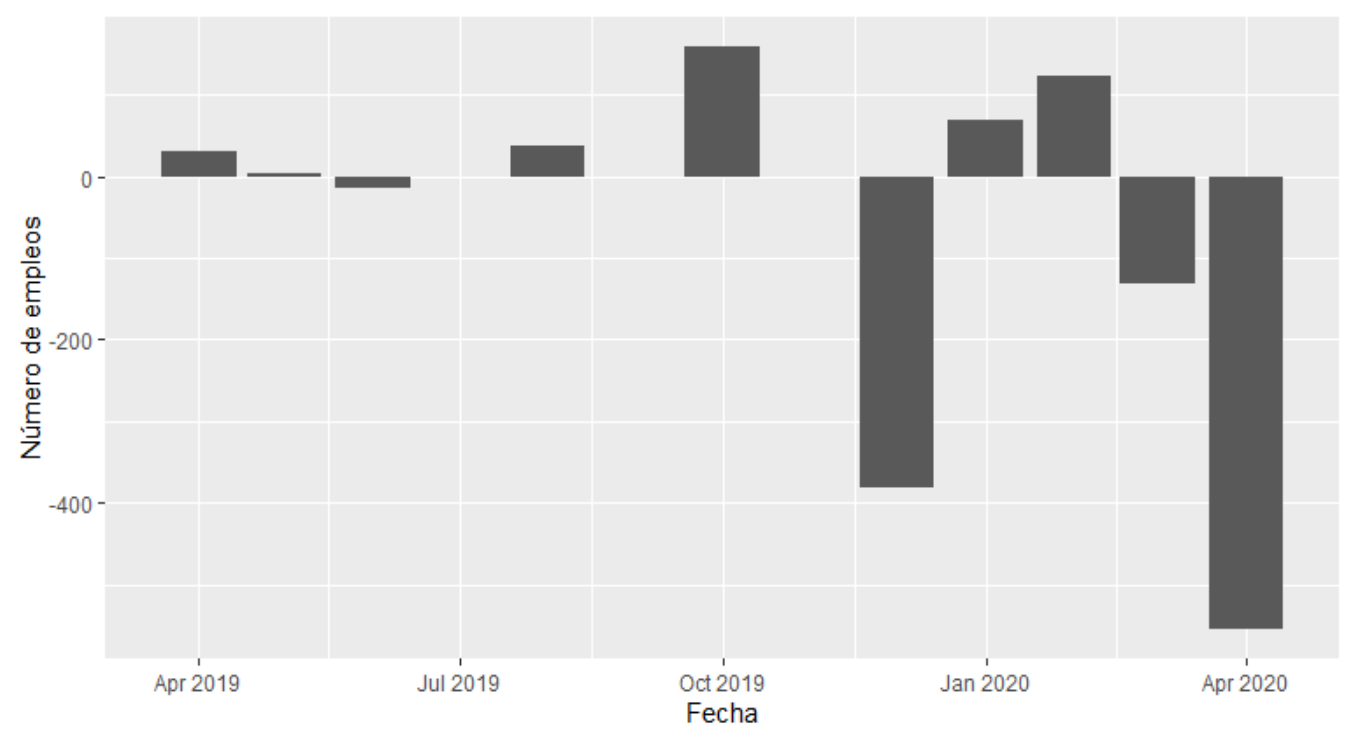

Figura 4. Variación del desempleo en 2019 y 2020.

La Figura 5 A) muestra la evolución de las remesas desde final del primer trimestre del año 2019 hasta el primer trimestre de 2020. Se observa una curva casi constante con alzas y bajas muy suaves hasta el primer trimestre de 2020. Durante este trimestre, la cuarentena aplicada en casi todos los países impactó en los empleos y su efecto lo perciben los países con economías débiles. A saber, el incremento histórico del 4.4\%, en la tasa de desempleo de Estados Unidos aunado a la dependencia económica hacia las remesas, afecta fuertemente a la economía mexicana (Expansión, 2020). No obstante, al inicio del segundo trimestre, se observó la reapertura progresiva de empresas en diversos sectores (El Economista, 2020b), lo que explica el repunte de las remesas.

Enseguida, la Figura 5 B) analiza la evolución de la inflación en México. Se observa que, desde enero del 2019, la inflación ha ido bajando hasta estabilizarse en diciembre del mismo año a una tasa inferior al 3\%. La pandemia del COVID-19 declarada en enero provocó un alza en la inflación de 3.7\% en febrero que se explica por la especulación provocada por el inicio de pánico en las diferentes actividades e incertidumbre sobre la pandemia. Después, durante marzo y abril se presentó una dura caída hasta llegar a 2.1\%, provocado por una reducción drástica de la demanda agregada de los productos. El aumento del desempleo, el cierre de los negocios y la reducción de la movilidad explican la tendencia de la demanda. En este caso productos básicos tienden a disminuir de precio. 


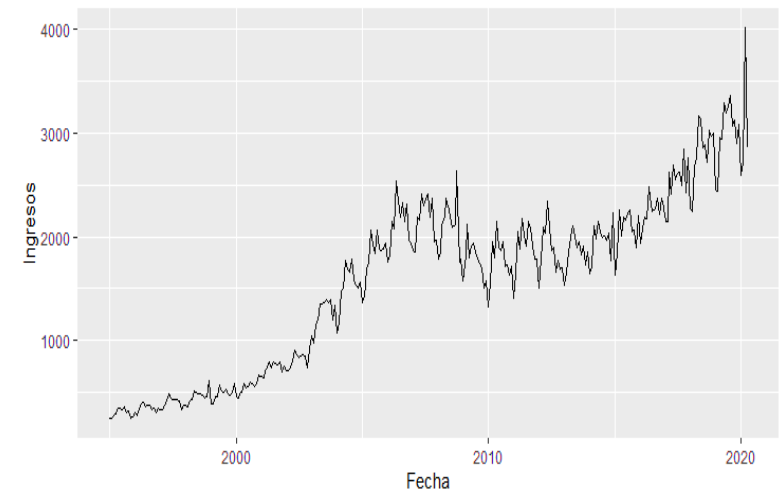

Figura 5 A): Evolución de las remesas

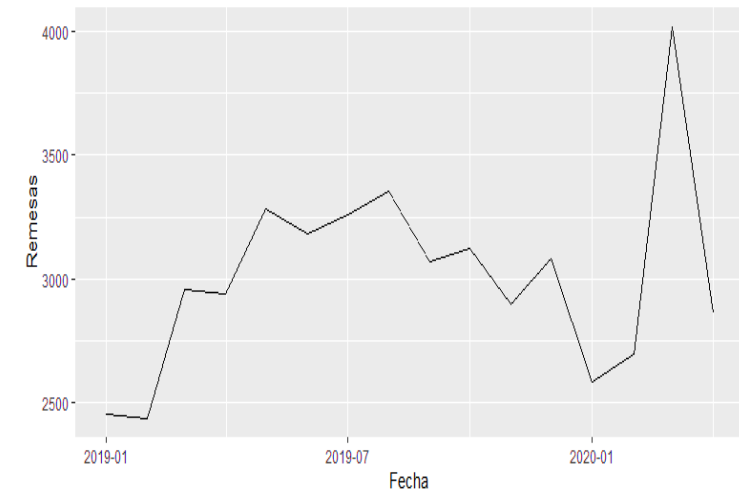

Figura 5 B): Evolución de la inflación

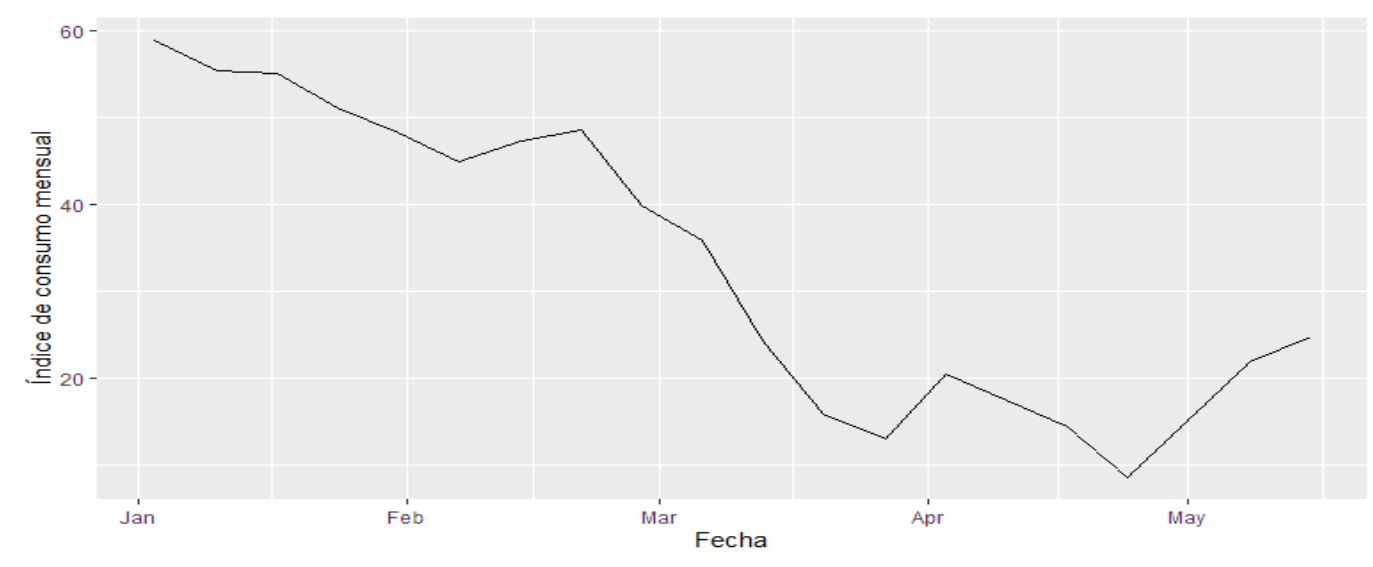

Figura 5 C): Índice de Consumo Mensual (enero-marzo, 2020)

Figura 5. Gráfica de remesas, inflación y consumo mensual. Fuente: Elaboración propia con datos de Banco de México (2020e), Banco de México (2020d) y Statista (2020a).

Finalmente, la Figura 5 C) presenta la capacidad de adquisición de los consumidores. Como se observa en la gráfica, la cuarentena y la pérdida de empleos han disminuido la capacidad de compra de la población. De enero a febrero hubo una desaceleración lenta al pasar de 58.68 a 48.47. La caída drástica comienza desde el punto señalado anteriormente hasta marzo 27 alcanzando 13.01. La situación sigue deteriorándose hasta llegar a 8.53 en abril 24. El aumento del desempleo, el cierre de miles de empresas, específicamente las nano, micro y pequeñas empresas (NAMYPES) y la poca movilidad por confinamiento explican la pérdida del poder adquisitivo de los consumidores. 


\section{Medidas para combatir el COVID-19 en México}

México atraviesa una de las peores crisis humanitarias, sociales y económicas en la historia reciente, que evoluciona continuamente. Por eso el gobierno desarrolló medidas para combatir la pandemia. Al final del primer trimestre y a principios del segundo, el gobierno aplicó una serie de medidas que se mencionan a continuación.

La primera etapa fue al final del primer trimestre, específicamente el 20 de marzo de 2020

- Plan 1: medidas de acopio de recursos. Infraestructura de salud (INSABI, IMSS e ISSSTE).

- Plan 2: Plan DN-III. Trabajo conjunto con las fuerzas armadas y su infraestructura sanitaria.

- La segunda etapa fue en el primer mes del segundo trimestre, específicamente el 16 abril 2020 según El Economista (2020c).

- Envío de cuatro meses de apoyo a adultos mayores.

- Otorgar tandas para el bienestar a los que viven al día.

- Aumento del presupuesto para fortalecer el blindaje de los programas sociales, en 622 mil 556 millones de pesos.

- Construcción del aeropuerto de Santa Lucia, Tren Maya y la refinería de Dos Bocas.

- Otorgar 2 millones 100 mil créditos a trabajadores formales e informales. Se otorgarán 3 millones de créditos dirigidos a la población más necesitada y a la clase media.

- Aumentar la rigidez en la austeridad republicana para lo que se reducirá el sueldo de funcionarios de alto nivel y se eliminarán aguinaldos, desde subdirectores hasta el presidente.

- Cancelación de diez subsecretarías. Se garantiza el empleo, con el mismo rango y salario, a quienes dejarán dichos cargos.

- Extender la suspensión de labores en áreas de gobierno, con goce de sueldo, hasta el 1 de agosto.

- Dejar de rentar edificios, vehículos, bodegas e inmuebles, entre otros ahorros.

- Creación de 2 millones de nuevos empleos. 
- Apoyo a 2.1 millones de micros y pequeños empresarios, entrega de 4 millones de créditos, lo que significa 307 mil 557 millones de pesos.

\section{Materiales y Métodos}

Los modelos basados en econometría y series de tiempo suelen utilizarse con frecuencia en el estudio de fenómenos económicos. Acorde a Montgomery, Zarnowitz, Tsay y Tiao (1998), la selección del modelo es un elemento clave en el momento de realizar pronósticos en economía. Entre estos modelos, los promedios móviles autorregresivos integrados o ARIMA por sus siglas en inglés suelen dar buenos resultados a pesar de que pueden ser más simples que otros. Los modelos ARIMA no proporcionan una explicación del fenómeno a estudiar, su principal utilidad radica en su efectividad para hacer predicciones a corto plazo. No obstante, se pueden obtener las componentes clásicas de una serie de tiempo, es decir, tendencia, estacionalidad, etc., a partir del modelo (Espasa y Peña 1990). Nyoni (2019), utilizaron métodos ARIMA para pronosticar el INPC anual de Bélgica desde 1960 hasta 2017. Previamente, Stock y Watson (1999) compararon los pronósticos lineales y no lineales de la tasa de inflación de Estados Unidos y realizaron pronósticos de la tasa de inflación de precios en los Estados Unidos, medidos por la tasa de inflación porcentual del INPC.

Dentro de los principales modelos lineales se encuentran los ARIMA (Mauricio, 2007). Este método ha sido aplicado exitosamente para predecir la tasa de desempleo e inflación y en otras áreas de las ciencias sociales (Montgomery, et al., 1998; Keenan y Stevens, 2016). Para series de tiempo que presentan estacionalidad, es decir, en las cuales la variabilidad de la serie depende del momento del año, se proponen modelos ARIMA estacionales.

El modelo general que permite predecir el valor de una variable $\mathrm{Y}$ en el tiempo $t+1$ en función del valor de la serie y del error del modelo $\varepsilon$ en el período actual $t$ y en los periodos tiene la siguiente forma (Yafee y McGee, 2000):

$$
X_{t+1}=f\left(t, X_{t}, X_{t-1}, \ldots, \varepsilon_{t}, \varepsilon_{t-1, \ldots}\right)
$$


Si la serie de tiempo tiene una parte determinista, se suele agregar dentro del modelo. Esto es conocido como drift. Un modelo $\operatorname{ARIMA}(p, q, d)(P, Q, D)_{s}$ con orden de estacionalidad $s$ y con drift toma la siguiente forma:

$$
\Phi_{P}\left(L^{S}\right) \phi_{p}(L)\left(1-L^{S}\right)^{D}(1-L)^{d}\left(X_{t}-\alpha-\beta t\right)=\theta_{q}(L) \Theta_{Q}\left(L^{S}\right) \varepsilon_{t},
$$

donde $\Phi_{P}, \phi_{p}, \theta_{q}, \Theta_{Q}$ son polinomios de grado $P, p, q$ y $Q$, respectivamente, $L^{k} X_{t}=X_{t-k}$, y $\varepsilon_{t}$ es el error al tiempo $t$. El modelo general se puede descomponer en las siguientes partes:

- $\phi_{p}(L)$ es la parte autorregresiva,

- $\Phi_{P}\left(L^{S}\right)$ es la parte autorregresiva estacional,

- $\left(1-L^{S}\right)^{D}$ es la parte integrada de la estacionalidad,

- $(1-L)^{d}$ es la parte integrada,

- $\left(X_{t}-\alpha-\beta t\right)$ es el drift aplicado a la serie estudiada,

- $\theta_{q}(L)$ es la parte correspondiente a los promedios móviles, y

- $\Theta_{Q}\left(L^{S}\right)$ son los promedios móviles aplicados a la estacionalidad.

Se enfatiza en que los coeficientes del modelo ARIMA pueden reescribirse de diferentes formas, según el contexto del fenómeno a modelar.

Además, se utilizarán diversos métodos estadísticos para estudiar las series de tiempo, a saber, correlogramas y correlogramas parciales para estudiar la autocorrelación en la serie, transformaciones de Box-Cox para tratar series no estacionarias en la varianza, la prueba de Dickey-Fuller aumentada para estudiar si la serie es o no estacionaria, el estimador AIC (Akaike information criterion) para elegir el modelo y la prueba de Ljung-Box sobre los residuales para validar los modelos (Nyoni, 2019). Por último, se utiliza el modelo para realizar los pronósticos de las series de tiempo. Los pronósticos obtenidos son mostrados con intervalos de confianza de $80 \%$ y 95\%. Para los cálculos se utilizó el software libre R (The R Project for Statistical Computing). 


\section{Resultados y Discusión}

Se estimaron el PIB, el INPC, la tasa de desempleo, las remesas, la inflación y el índice de consumo mensual, para comprender el impacto del COVID-19 en la economía mexicana al cierre del año 2020. Para el análisis de las series de tiempo que aparecen en este trabajo se procedió de la siguiente forma:

1) Para el caso del INPC, desempleo e inflación, se utilizan los siguientes modelos. Nyoni (2019) para el caso del INPC, Montgomery et al. (1998) para estudiar el desempleo y Stock y Watson (1999) para el pronóstico de la inflación.

2) Para el PIB, las remesas y el ICM se determinan los modelos de la siguiente forma. Primero se aplicó una prueba de Dicke-Fuller aumentada para ver si la serie es o no estacionaria. A partir de la gráfica de la serie original, de los correlogramas y de los correlogramas parciales se decidió si la serie necesita una transformación de BoxCox, si se debe restar la tendencia (drift) y si tiene estacionalidad. En respuesta a lo que se resolvió en el punto anterior se aplican las diferencias en los casos de presentar estacionalidad o de no ser estacionarias, y se ajusta la recta tangente y se resta al modelo. Después se analizan los correlogramas y correlogramas parciales para proponer modelos para, finalmente, escoger el mejor candidato usando el estimador AIC.

En ambos casos se validan los resultados analizando los residuales de forma visual con el correlograma y un histograma, y una prueba de Ljung-Box sobre la correlación en los residuales. En la Tabla 1 se muestran los modelos utilizados en las diferentes series de tiempo. Para un entendimiento más profundo sobre los modelos, sus ecuaciones y significados puede consultarse (Espasa y Peña, 1990). 
Tabla 1.

Modelos ARIMA aplicados para el análisis del PIB, el INPC, la tasa de desempleo, las remesas, la inflación y el índice de consumo mensual

\begin{tabular}{lc}
\hline \multicolumn{1}{c}{ Modelo } & Ecuación \\
\hline ARIMA $(0,2,1)$ & $X_{t}=\mu+X_{t-1}+\theta_{1} \varepsilon_{t-1}+\varepsilon_{t}$. \\
ARIMA $(4,0,0)$ & $X_{t}=\mu+\phi_{1} X_{t-1}+\phi_{2} X_{t-2}+\phi_{3} X_{t-3}+\phi_{4} X_{t-4}+\varepsilon_{t}$ \\
ARIMA $(1,0,0)$ & $X_{t}=\mu+\phi_{1} X_{t-1}+\varepsilon_{t}$ \\
ARIMA $(1,1,2)(1,1,1)_{12}$ & $\left(1+\Phi_{1} L^{12}\right)\left(1+\phi_{1} L\right)\left(1-L^{12}\right)(1-L)^{1} X_{t}=\left(1+\theta_{1} L+\theta_{2} L^{2}\right)\left(1+\Theta_{1} L^{12}\right) \varepsilon_{t}$, \\
ARIMA $(1,0,2)(0,1,1)_{4}$ & $\left(1+\phi_{1} L\right)\left(1-L^{4}\right)\left(X_{t}-\alpha\right)=\left(1+\theta_{1} L+\theta_{2} L^{2}\right)\left(1+\Theta_{1} L^{4}\right) \varepsilon_{t}$, \\
con drift & \\
\hline
\end{tabular}

En la Figura 6 muestra el pronóstico del PIB para lo que resta del 2020 y el 2021. La serie de tiempo del PIB muestra tendencia (drift) y estacionalidad. El modelo $\operatorname{ARIMA}(1,0,2)(0,1,1)_{4}$ con drift es el que mejor ajusta los datos. Se pronostica que la tendencia continúe para los siguientes trimestres, aunque frenada ligeramente por la caída en el primer trimestre del 2020.

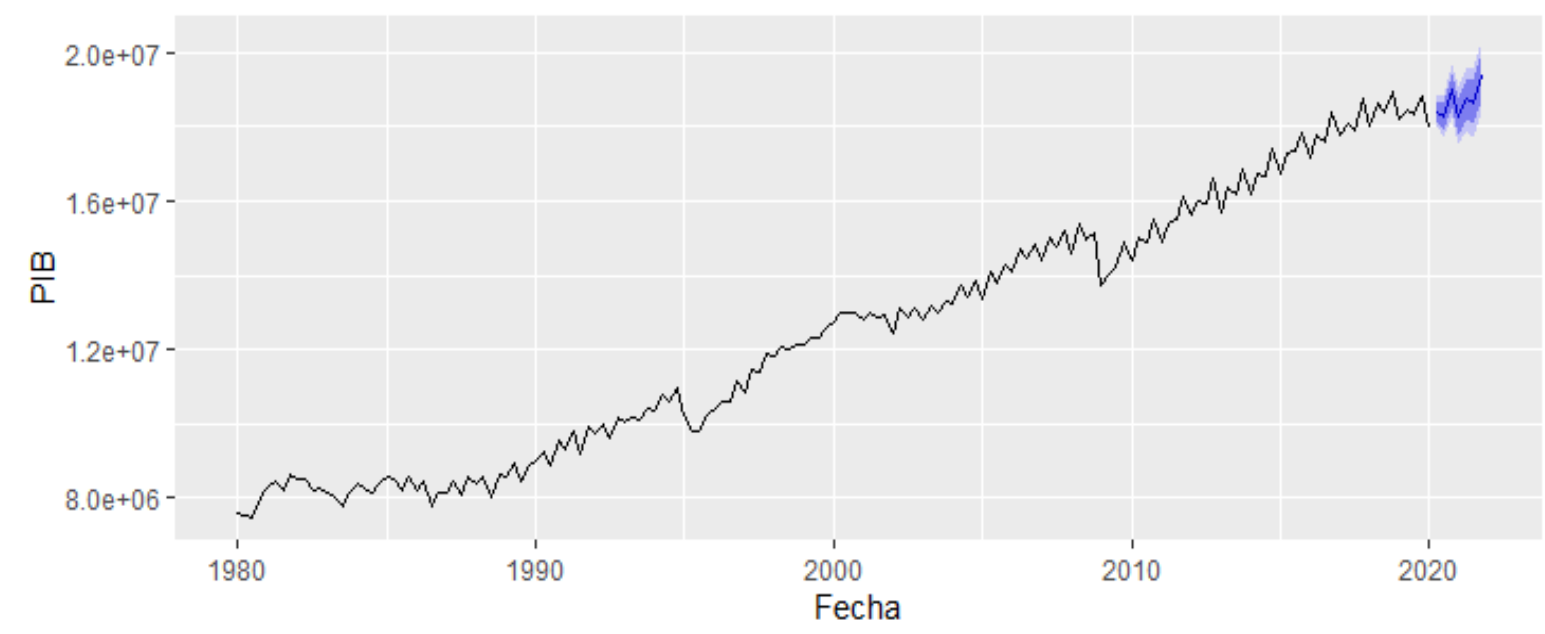

Figura 6: Pronóstico de las PIB. Fuente. Elaboración propia con los resultados del modelo $\operatorname{ARIMA}(1,0,2)(0,1,1)_{4}$.

En relación con el Índice Nacional de Precios al Consumidor (INPC), se muestra la serie de datos históricos en la Figura 7. Para el pronóstico, se utilizó un modelo $\operatorname{ARIMA}(0,2,1)$ para predecir el comportamiento del INPC. Se pronostica que el INPC disminuirá al continuar el 
año, continuando con lo que se ha estado observando en los periodos anteriores (ver Figura 2).

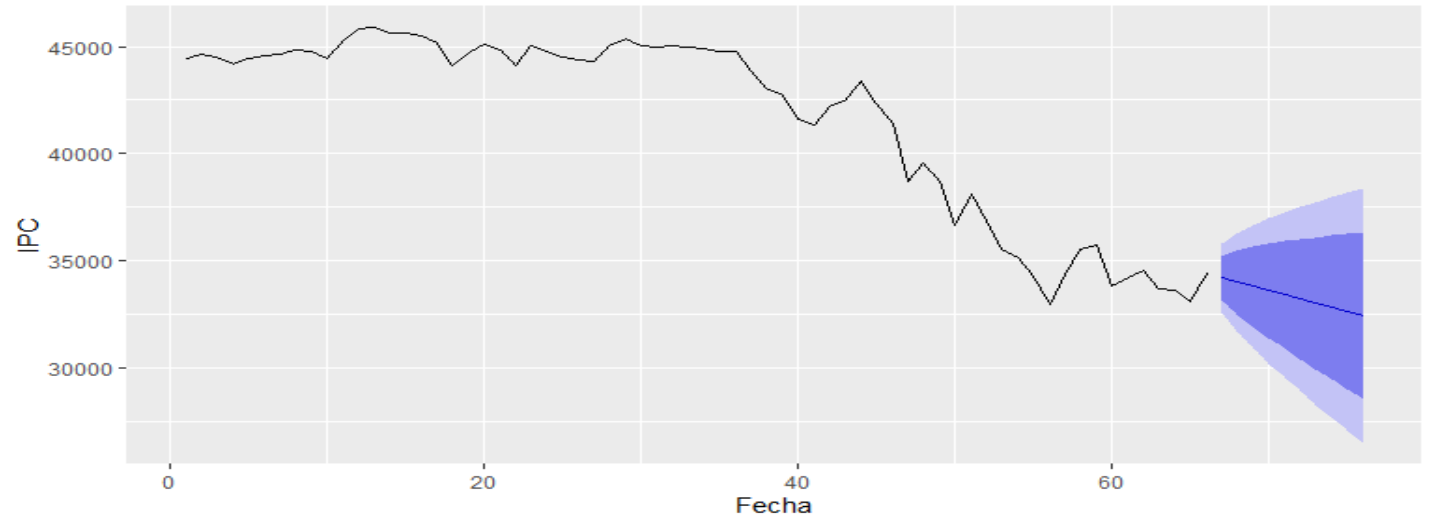

Figura 7. Pronóstico para el Índice Nacional de Precio al Consumidor (INPC) al cierre de 2020. Fuente. Elaboración propia con los resultados del modelo $\operatorname{ARIMA}(0,2,1)$.

Por otra parte, el desempleo fue analizado utilizando datos de dos fuentes. Primero, se obtuvieron los datos anuales de World Bank (2019), ver Figura 8. Segundo, de Flores (2020) que a su vez es información del Instituto Mexicano del Seguro Social (IMSS) se obtuvieron datos de variación del empleo en el primer cuatrimestre de 2020 (Figura 9).

Para el caso de los datos anuales, se resalta el cambio abrupto en el comportamiento del desempleo en el año 2020 con el surgimiento del COVID-19. Se utilizó el modelo $\operatorname{ARIMA}(4,0,0)$. En este modelo, el valor a predecir depende fuertemente de los cuatro valores anteriores. Por lo tanto, en este caso se observa que el pronóstico estará alrededor del valor 3.5 .

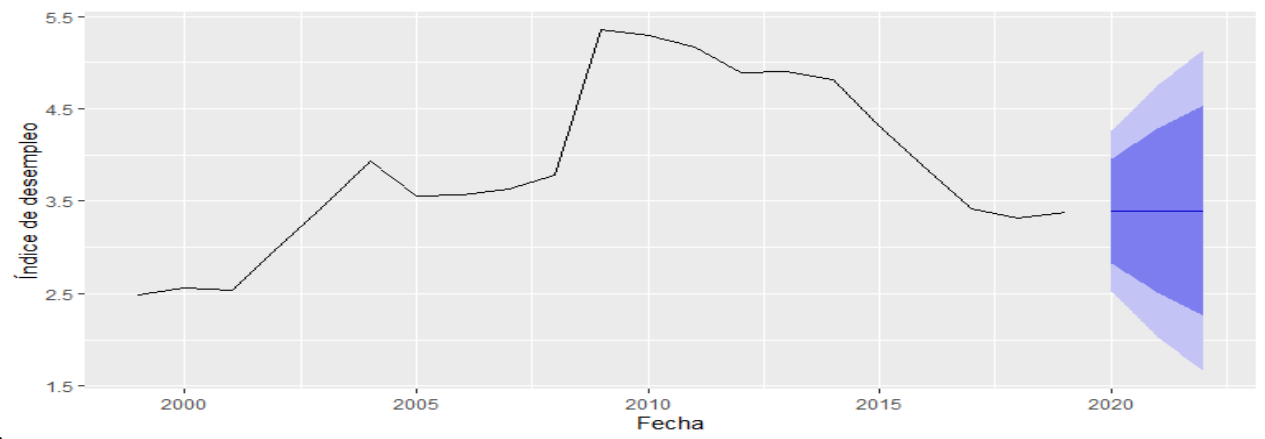

Figura 8. Pronóstico para Índice de desempleo al cierre de 2020. Fuente. Elaboración propia con los resultados del modelo ARIMA $(4,0,0)$. 
Con relación a la variación del empleo en el primer cuatrimestre de 2020, la gráfica de la Figura 9 muestra que la cantidad de empleos ha bajado en el transcurso del año, contrario a lo que se predice con los datos anteriores a la pandemia.

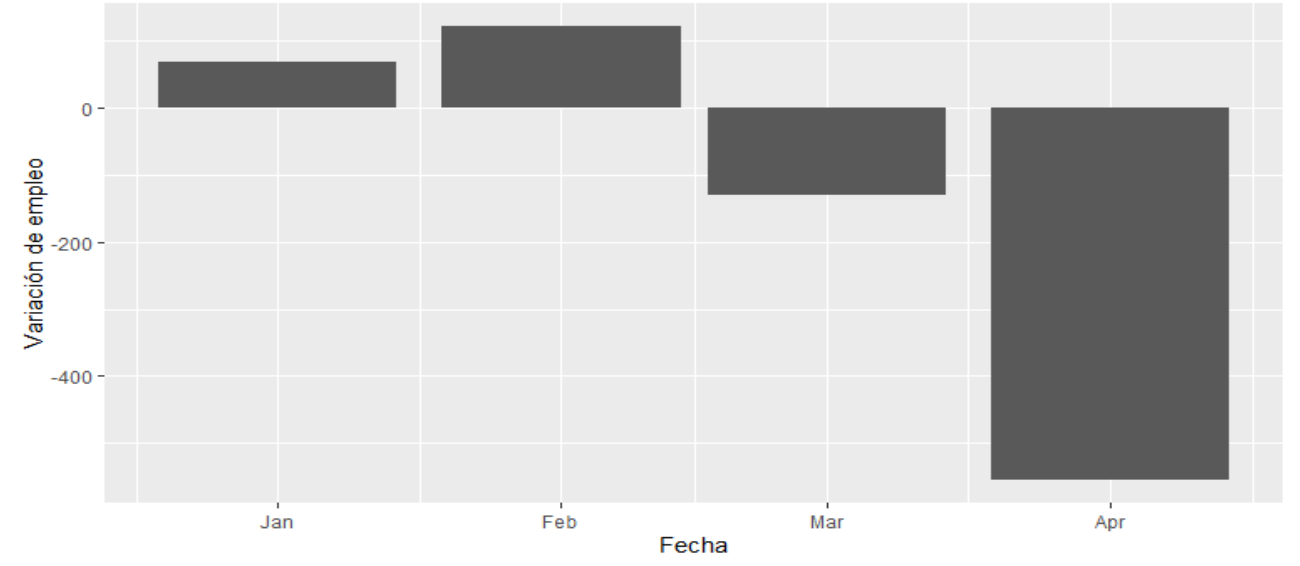

Figura 9. Variación del empleo en el primer cuatrimestre de 2020. Fuente: Elaboración propia.

El modelo que se utilizó para el pronóstico de las remesas es $\operatorname{ARIMA}(1,1,2)(1,1,1)_{12}$, ver Figura 10. Se observa que la cantidad de ingresos debido a las remesas tenderá a aumentar en lo que resta del año y en el año siguiente.

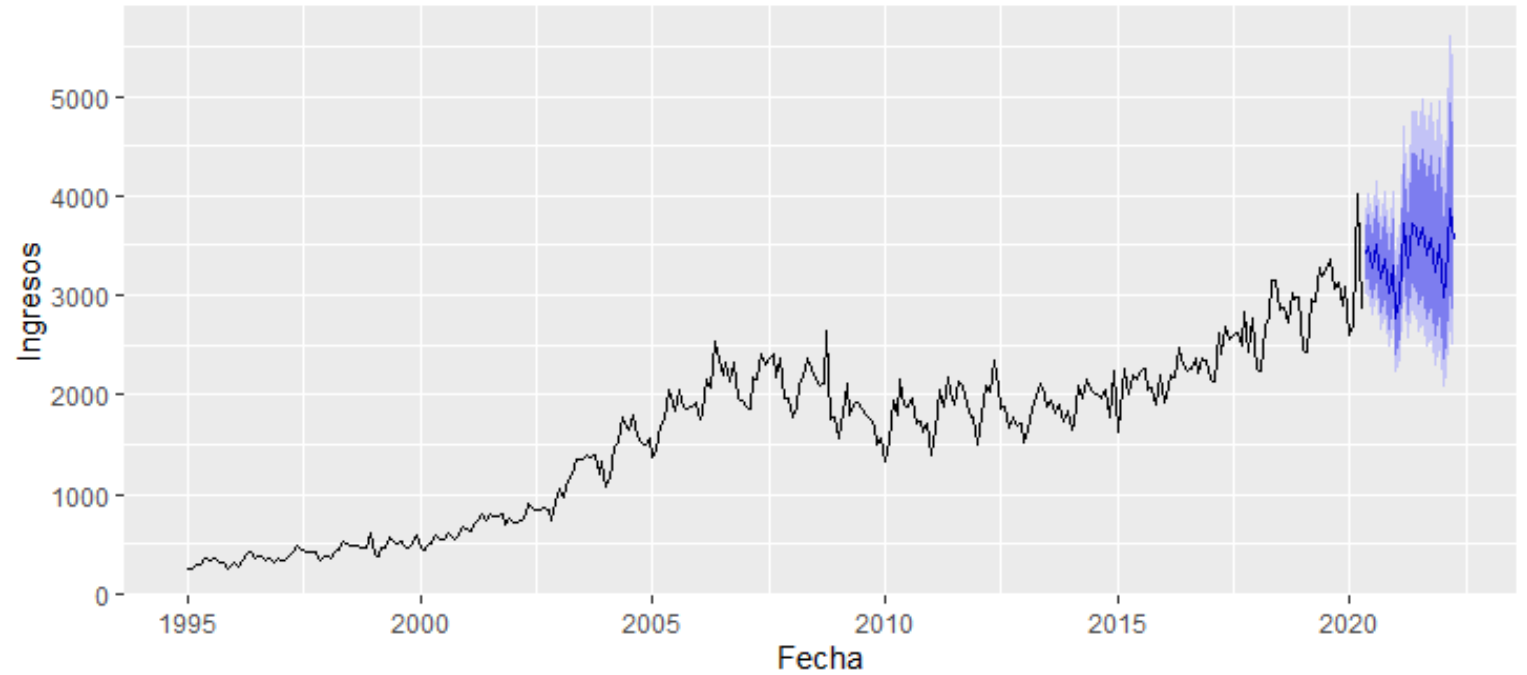

Figura 10. Pronóstico para Índice de desempleo al cierre de 2020. Fuente. Elaboración propia con los resultados del modelo ARIMA $(1,1,2)(1,1,1)_{12}$.

Para el análisis y pronóstico de la inflación se utilizó un modelo ARIMA (4,0,0). La Figura 11 muestra el pronóstico de la inflación para los siguientes meses del 2020. Se puede observar 
una pequeña variabilidad en la inflación en el resto del año. Se espera que el valor de la tasa de inflación se encuentre entre 3.0 y 3.5 al cierre del año 2020.

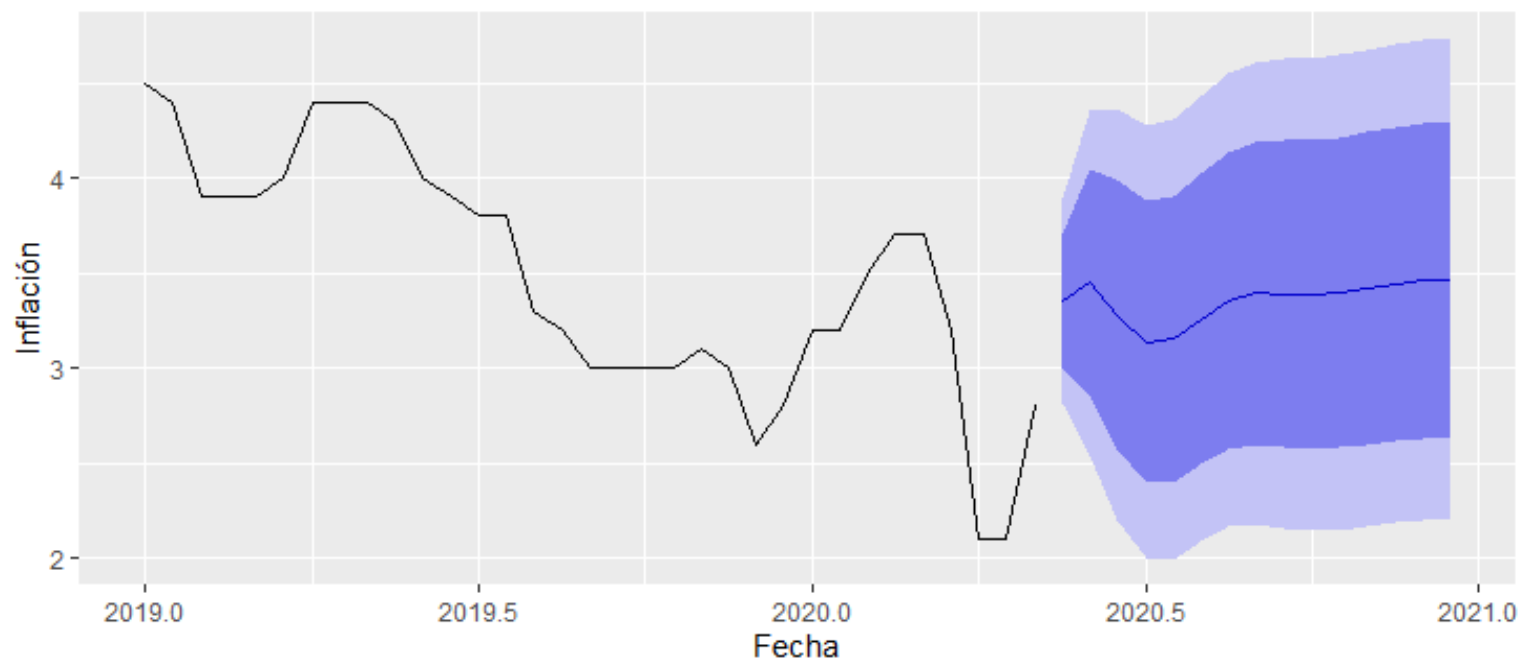

Figura 11. Pronóstico para la inflación al cierre de 2020. Fuente. Elaboración propia con los resultados del modelo ARIMA $(4,0,0)$.

En el análisis de la serie de tiempo de ICM se muestra que el modelo ARIMA(1,0,0), el cual es el modelo de autorregresión más simple, se ajusta bien a los datos. A partir de este modelo se pronostica que el ICM aumente lentamente durante el resto del año para estabilizarse cerca del valor 40, esto después de una fuerte caída al principio del 2020.

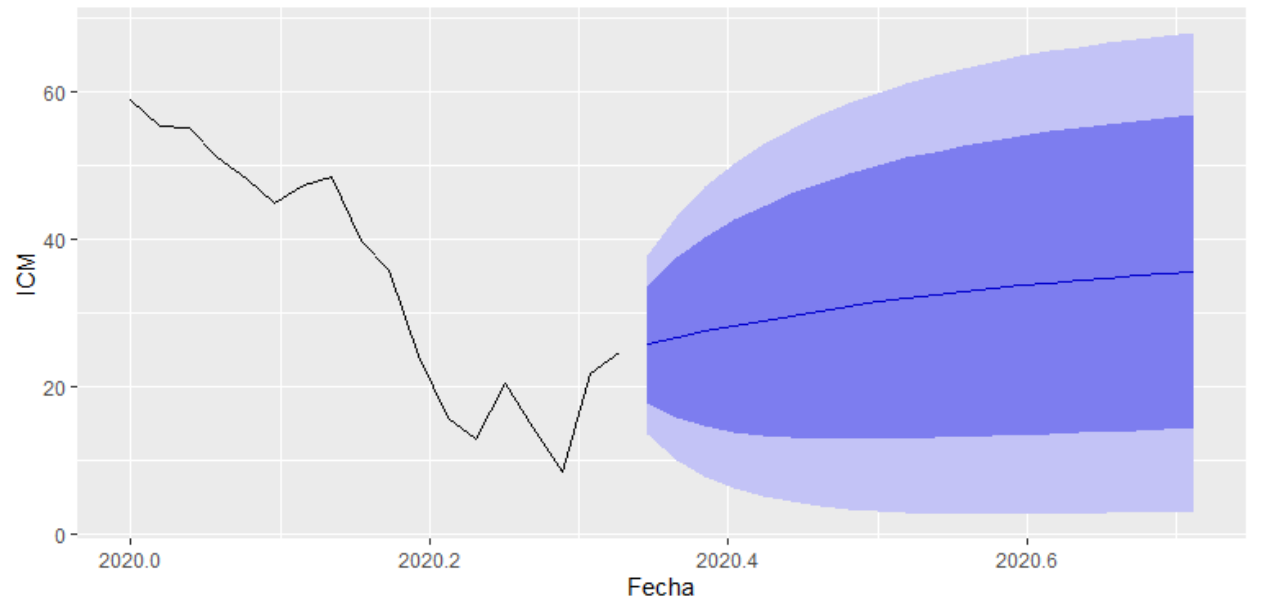

Figura 12. Pronóstico para el ICM al cierre de 2020. Fuente. Elaboración propia con los resultados del modelo $\operatorname{ARIMA}(1,0,0)$.

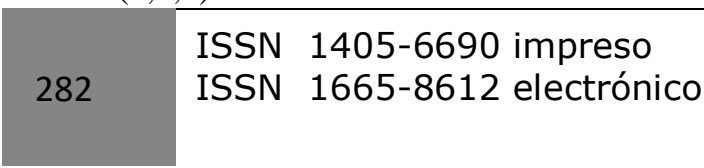


Después de la pandemia se espera que el IPC aumente hasta el último trimestre del año en curso porque se espera un aumento en la demanda, principalmente productos de la canasta básica (compuesto por productos de importación) por lo que es importante que el gobierno aplique una política fiscal expansiva que implica el aumento del gasto público y la reducción de la recaudación fiscal. Esto permitirá reactivar la economía, a través de un aumento de la producción, el aumento del consumo y el empleo.

Los efectos de la crisis han sido duros principalmente en el sector del empleo. Entre los meses de marzo y mayo se perdieron cerca de un millón de empleos y muchas empresas se declararon en quiebra. Las empresas más afectadas son las MyPES ya que no cuentan con el capital suficiente para enfrentar una crisis de esta magnitud. El otorgamiento de crédito a esas empresas no se hizo de forma adecuada por lo que la cantidad de empresas inactivas tenderá al alza en el transcurso del año.

En otras palabras, al paro de actividades las MyPES (incluso las medianas) no pudieron mantener en la nómina a todo su personal. Esto tiene lógica, pues al caer las ventas, caen los ingresos y las empresas no pueden hacer frente a sus compromisos de pago de salarios y deciden despedir a los trabajadores o reducir su plantilla significativamente. Los trabajadores desempleados pierden su única fuente de ingresos y aunado al factor psicológico deciden comprar poco (lo indispensable. es decir, alimentos) y en un escenario así la economía del país se detiene. La producción baja, la demanda se incrementa. Como resultado los precios suben.

Lo anterior justifica que la tasa de inflación se encontrará entre 3.0 y 3.5 al cierre del año 2020, es decir que se observará un aumento comparable a abril y mayo. Esto se explica por la apertura progresiva de los diferentes sectores de la economía, principalmente los productos importados. La preocupación es la variación del tipo de cambio con respecto al dólar que encarece los productos que vienen de fuera y que se encuentran en la canasta básica de bienes y servicios (incremento sobre todo de alimentos, bebidas y medicamentos) representativa del consumo final de los hogares.

El ICM tenderá a aumentar durante las siguientes semanas por la reapertura progresiva de las actividades económicas. 


\section{Conclusiones}

Los impactos macroeconómicos a nivel global de la pandemia de COVID-19 se registran en casi todos los sectores, las cifras son preliminares por lo que el costo final debe determinarse. De ahí la necesidad de estudiar algunas variables económicas como el crecimiento del PIB, el IPC, la tasa de cambio del peso mexicano frente al dólar, la tasa de desempleo, las remesas, la inflación y el ICM.

Se realizaron pronósticos de series de tiempo usando modelos ARIMA. Se vislumbra que el IPC seguirá decreciendo para lo que resta del año. Por un lado, el pronóstico la tasa de desempleo permanecerá en el valor de $3.38 \%$, por el otro se observa que el desempleo aumenta. Las remesas continuarán alrededor de los 2 mil 800 millones de dólares. La inflación se acercará al valor de 3.5 a finales del año. Por último, el ICM continuará creciendo lentamente durante la segunda mitad del 2020.

Se mostraron las medidas adoptadas por el gobierno mexicano para disminuir el impacto de la pandemia en la economía mexicana, por ejemplo, el apoyo a adultos mayores, la construcción del aeropuerto de Santa Lucia, el Tren Maya y la refinería de Dos Bocas, la cancelación de diez subsecretarías, el apoyo a 2.1 millones de MyPES. A partir de los pronósticos realizados y tomando en cuenta otros factores como las obras públicas mencionadas anteriormente, el fortalecimiento del peso mexicano, la recuperación del precio del petróleo, el aumento del ingreso tributario petrolero al mes de diciembre derivado de la contratación de coberturas.

No se puede visualizar por el momento las repercusiones de las acciones del gobierno en la economía nacional. Sin embargo, se prevé un escenario desfavorable por la tendencia que muestran los factores analizado. A manera de ejemplo, entre marzo y mayo se perdieron cerca del millón de empleos y el quiebre de muchas empresas, particularmente de MyPES que no cuentan con el capital suficiente para enfrentar una crisis de esta magnitud. Cabe resaltar que la principal limitación a esta investigación es la falta de datos por ser un suceso peculiar con escaso tiempo de surgimiento, por lo que son resultados preliminares. De lo anterior desarrollado, el gobierno debe aplicar una política fiscal expansiva que implique el aumento del gasto público y la reducción de la recaudación fiscal. El aumento de programas 
sociales, la reducción de los impuestos y la creación de empleos ayudarán a estimular el consumo en los hogares y por supuesto la economía nacional.

Finalmente, se presentan dos recomendaciones para mitigar los efectos de la pandemia.

- $\quad$ Aumentar el presupuesto.

La participación del gobierno con respecto al aumento del gasto público durante la pandemia ha sido insuficiente comparada con los países de la región. Por ejemplo, Perú invirtió el 9\% de su PIB mientras que México el 1\% (Perfil, 2020). En el caso del reparte de crédito a las MyPES (25,000 pesos) éste es insuficiente para gastos de renta, salarios y otros. Según Dossier Político (2020), el programa, planteó la entrega de casi 4 millones de créditos, que significarían una inversión de 307 mil 557 millones de pesos.

Dado que la pandemia ha traído nuevos cambios en el funcionamiento de las MyPES, según lo señala Peralta (2020), éstas deben ajustar sus procesos y formas de concebir sus relaciones comerciales. Lo esencial es reflexionar acerca de lo que genera valor en su organización para conservar los clientes y garantizar su sobrevivencia.

- Anticipar y organizar la respuesta

Refiriéndose a la respuesta del gobierno a esta emergencia, se menciona nuevamente el caso del programa de crédito a las MyPES. Como lo menciona Dossier Político (2020) casi un mes después de que el coronavirus fuera denominado como pandemia, tanto Economía como el IMSS lanzaron estos apoyos: "ya había ahogados cuando buscaron los salvavidas", se hizo de manera apresurada a pesar de que tuvieron tiempo. Con relación a los créditos otorgados, hasta el 15 de mayo solo se habían entregado 740 mil, que significa recursos por 44 mil 721 millones de los 307 mil 557 millones de pesos asignados. 


\section{Referencias}

Baldwin, R. y Di Mauro, B. (2020). Economics in the Time of COVID-19. CEPR Press, London. Recuperado en: https://voxeu.org/system/files/epublication/COVID-19.pdf

Banco de México. (2020a). Forecasted gross domestic product (GDP) growth in Mexico in 2020, by month of forecast [Graph]. Statista. Retrieved June 03, 2020, from https://s443-www-statista-com.lasalle.lsproxy.net/statistics/1108139/expected-gdpgrowth-mexico/

Banco de México. (2020b). Serie histórica diaria del tipo de cambio peso-dólar - (CF373).

Recuperado

en:

https://www.banxico.org.mx/SieInternet/consultarDirectorioInternetAction.do?secto $\underline{\mathrm{r}=6 \& \text { accion }=\text { consultarCuadro \&idCuadro }=\mathrm{CF} 373 \& \text { locale }=\mathrm{es}}$

Banco de México. (2020c). Balanza de pagos, Remesas. Recuperado de https://www.banxico.org.mx/SieInternet/consultarDirectorioInternetAction.do?accio $\underline{\mathrm{n}=\text { consultarCuadro\&idCuadro }=\mathrm{CE} 81 \& \text { locale }=\mathrm{es}}$

Banco de México. (2020d). Expectativas de Inflación para los Próximos 12 Meses - (CR155).

Recuperado de https://www.banxico.org.mx/SieInternet/consultarDirectorioInternetAction.do?accio $\underline{\mathrm{n}=\text { consultarCuadro\&idCuadro }=\mathrm{CP} 154 \& \text { locale }=\mathrm{es}}$

Banco de México. (2020e). Ingresos por Remesas - (CE81). Recuperado de https://www.banxico.org.mx/SieInternet/consultarDirectorioInternetAction.do?secto $\underline{\mathrm{r}=1 \& \text { accion }=\text { consultarCuadro\&idCuadro }=\mathrm{CE} 81 \& \text { locale }=\mathrm{es}}$

Barua, S. (2020). Understanding Coronanomics: The economic implications of the coronavirus (COVID-19) pandemic. SSRN. Recuperado en: http://dx.doi.org/10.2139/ssrn.3566477

Comisión Económica para América Latina y el Caribe (CEPAL) (2020). América Latina y el Caribe ante la pandemia del COVID-19 Efectos económicos y sociales. Naciones 
Unidas.

Recuperado

en:

http://www.pued.unam.mx/export/sites/default/archivos/covid/CEPAL.pdf

Dossier Político (2020). ¿Por qué la entrega de los créditos de AMLO tiene un avance menor

a 25\%? Recuperado de

https://www.dossierpolitico.com/vernoticias.php?artid=234134\&relacion=dossierpo $\underline{\text { litico\&categoria }=2}$

Desempleo de Estados Unidos, (2020). Expansión. Recuperado de https://datosmacro.expansion.com/paro/usa?sc=LAB-

Juarez, E. (21 de abril de 2020). Citibanamex anticipa una caída del PIB de México de hasta 9\% en 2020. El Economista. https://www.eleconomista.com.mx/economia/Citibanamex-anticipa-una-caida-delPIB-de-Mexico-de-hasta-9-en-2020--20200421-0069.html

Reuters (18 de mayo de 2020). Casi 50 Estados iniciaron reapertura este lunes; autoridades alivian $\quad$ restricciones. $\quad E l \quad$ Economista. https://www.eleconomista.com.mx/internacionales/Casi-50-estados-de-EUiniciaron-reapertura-este-lunes-autoridades-alivian-restricciones-20200518$\underline{0073 . h t m l}$

Pérez, M. (22 de abril de 2020) ¿Qué medidas presentó AMLO para enfrentar el Covid-19 y la caída del petróleo? El Economista. https://www.eleconomista.com.mx/politica/Que-medidas-presento-AMLO-paraenfrentar-el-Covid-19-y-la-caida-del-petroleo-20200422-0061.html

Espasa, A., y Peña, D. (1990). ARIMA models, the steady state of economic variables and their estimation. Documentos de trabajo/Banco de España, 9008.

Flores, Z. (2020) Se perdieron 753 mil empleos en mes y medio. El Financiero. https://www.elfinanciero.com.mx/economia/se-pierden-555-mil-empleos-formalesen-abril-suman-mas-de-685-mil-contando-marzo

Hevia, C. y Neumeyer, A. (2020). Un marco conceptual para analizar el impacto económico del COVID-19 y sus repercusiones en las políticas. PNUD LAC C19 PDS No. 1, Universidad Torcuato Di Tella. Recuperado 
https://www.undp.org/content/dam/rblac/Policy\%20Papers\%20COVID\%2019/UN DP-RBLAC-CD19-PDS-Number1-ES-final.pdf

INEGI. (2020a). Estimación oportuna del producto interno bruto en México durante el primer trimestre de 2020 (cifras desestacionalizadas). Comunicado de prensa núm. 195/20 30 de abril de 2020 página 1/2. Recuperado en: https://www.inegi.org.mx/contenidos/saladeprensa/boletines/2020/pib_eo/pib_eo20 20_04.pdf

INEGI. (2020b). Monthly unemployment rate in Mexico from January 2019 to March 2020 [Graph]. Statista. Retrieved June 03, 2020, from https://s443-www-statistacom.lasalle.lsproxy.net/statistics/1108285/mexico-monthly-unemployment-rate/

INEGI. (2020c). Indicadores de Ocupación y Empleo durante el primer trimestre de 2020. Cifras desestacionalizadas.

INEGI. (2020d). Producto Interno Bruto Cifras desestacionalizadas por grupo de actividades económicas. Recuperado de www.inegi.org.mx/temas/pib/default.html\#Informacion_general

Keenan A. y Stevens, J. (2016). Applied multivariate statistics for the social sciences. Routledge 6a. Ed. New York and London.

Kuckertz A., Brändle L., Gaudig A., Hinderer S., Morales-Reyes C., Prochotta A., Steinbrink K.M y Berger E. (2020). Startups in times of crisis- A rapid response to the COVID19 pandemic, Journal of Business Venturing Insights, 13, http://doi.org/10.1016/j.jbvi.2020.e00169

Lee J.W. y McKibbin, W.J. (2004a). Estimating the Global Economic Costs of SARS. In: Institute of Medicine (US) Forum on Microbial Threats; Knobler S, Mahmoud A, Lemon S, et al., editors. Learning from SARS: Preparing for the Next Disease Outbreak: Workshop Summary. Washington (DC): National Academies Press (US). Disponible en: https://www.ncbi.nlm.nih.gov/books/NBK92473/

Lee J. W. and McKibbin Warwick W. J., (2004b). Globalization and Disease: The Case of SARS, Asian Economic Papers, MIT Press 3(1), 113-131. 
Mauricio, J. A. (2007). Introducción al análisis de series Temporales. Madrid: Universidad Complutense de Madrid. Recuperado en: https://www.ucm.es/data/cont/docs/5182013-11-11-JAM-IAST-Libro.pdf

Mckibbin W., Anu C y Sidorenko A. (2006). Global Macroeconomic Consequences of Pandemic Influenza, Centre for Applied Macroeconomic Analysis. Recuperado en: https://cama.crawford.anu.edu.au/pdf/working-papers/2006/262006.pdf

Montgomery A., Zarnowitz V., Tsay R. y Tiao G. (1998). Forecasting the U.S. Unemployment Rate. Journal of the American Statistical Association, 93(442), 478493. http://dx.doi.org/10.1080/01621459.1998.10473696

Nyoni T. (2019). Time series modeling and forecasting of the consumer price index in Belgium, MPRA Paper 92414, University Library of Munich, Germany.

Organización Mundial de la Salud (OMS) (2020). Enfermedad por el Coronavirus (COVID19). Organización Mundial de la Salud, Recuperado en: https://www.paho.org/es/temas/coronavirus/enfermedad-por-coronavirus-covid-19

Organización de las Naciones Unidas (ONU) (2020), América Latina sufrirá la mayor recesión económica de su historia por el coronavirus. Recuperado de https://news.un.org/es/story/2020/04/1473192.

Peralta, G, J. (2020). Lo esencial y lo superfluo en la reactivación de México, Emprendedores, 184, 12-15. Recuperado en: http://emprendedores.unam.mx/articulo.php?id_articulo=686

Coronavirus: los países que más dinero invierten en combatir al Covid-19 (19 mayo, 2020). Perfil. $\quad$ https://www.perfil.com/noticias/internacional/coronavirus-los-paises-que$\underline{\text { mas-dinero-invierten-en-combatir-al-covid-19.phtml }}$

Powell, A. (2020). El coronavirus en América Latina y el Caribe y más allá. En Nuguer, Victoria y Powell, Andrew. Políticas para combatir la pandemia, Banco Interamericano de Desarrollo.

Change of the Consumer Price Index (CPI) in Mexico from January to March, 2019 and 2020 [Graph] (March 9, 2020a). Statista. Retrieved June 03, 2020, from https://s443-www- 
statista-com.lasalle.lsproxy.net/statistics/1107928/change-consumer-price-index$\underline{\text { mexico/ }}$

Mexico's IPC stock exchange index from January 2 to April 6, 2020 (in Mexican pesos) [Graph] (April 8, 2020ab). Statista. Retrieved June 03, 2020, from https://s443-wwwstatista-com.lasalle.lsproxy.net/statistics/1074108/mexico-ipc-stock-market-index/

Exchange rate of the Mexican peso to the U.S. dollar (USD) from February 17 to April 27, 2020 [Graph] (April 27, 2020c). Statista. Retrieved June 03, 2020, from https://s443Www-statista-com.lasalle.lsproxy.net/statistics/1108002/mexican-peso-exchangerate-usd/

Stock J. and Watson M. (1999). Forecasting Inflation. Journal of Monetary Economics, 44(2), 293-335. Recuperado en http://www.sciencedirect.com/science/article/pii/S0304-3932(99)00027-6

World Bank. (December 20, 2019). Mexico: Unemployment rate from 1999 to 2019. Statista. Retrieved June 03, 2020, from https://s443-www-statistacom.lasalle.lsproxy.net/statistics/263702/unemployment-rate-in-mexico/

Yafee, R. y McGee, M. (2000) Introduction to Time Series Analysis and Forecasting with Applications of SAS and SPSS. Primera edición, Accademic Press, Inc. 